\title{
New treatments for osteoarthritis must be based on evidence
}

Globally, osteoarthritis is one of the most common medical conditions, causing joint pain for millions of people. Trials of medical treatments for osteoarthritis seek to manage symptoms, primarily joint pain, and disease modification-ie, stopping progression or bring about disease modification [A: slightly repetitive now, could we remove the text in red? Perhaps changing to "eg," rather than "ie"?]. In some European countries, such as Germany, low-dose radiation therapy is used in routine clinical practice and believed by many to be effective for osteoarthritis; however, in the UK such therapy would be considered an experimental treatment. Following on from their previous two studies that did not meet their primary endpoints at 3 months, ${ }^{1,2}$ now in The Lancet Rheumatology, Cornelia van den Ende and colleagues report the follow-up results of these two randomised sham-controlled trials of low-dose radiation therapy in patients with hand and knee osteoarthritis. ${ }^{3}$ From these extended follow-up data, the authors suggest a lack of evidence for any clinically significant delayed response to treatment up to 1 year.

Whether studying novel or existing therapies, the literature over the past 20 years is littered with trials in osteoarthritis that did not show efficacy; why is this? Several topical considerations are taken into account in the present study, including the importance of careful study design, in this case including sham treatment. Placebo effects are evident in any painful condition, and osteoarthritis is no exception. Clinical practitioners who deliver interventions can easily believe that a treatment works because their patients feel better. Regression to the mean (in this context, pain improving over time) and magnitude of placebo responses correlating with the context-eg, invasiveness or perceived risk of treatments-mean that our clinical observations and assumptions about efficacy of treatments are often flawed. ${ }^{4}$ Such observations justify a need for further high quality, adequately powered, randomised placebo-controlled trials, and, highlight that our practice as clinicians is led by their findings. In another recent example, a large randomised controlled trial of hydroxychloroquine in hand osteoarthritis did not show efficacy over placebo for average hand pain over
12 months, despite many rheumatologists believing that it was effective. ${ }^{5}$ Some clinicians might feel they can select which patients might respond based on their own clinical judgement and experience, but none of these studies identified baseline features that were predictive of treatment outcomes.

Why does radiation not work in osteoarthritis? van den Ende and colleagues propose a potential mechanism of action could be by reduction of inflammation in soft tissue and particularly synovial inflammation seen in many patients with the condition. Synovial inflammation or effusion in osteoarthritis is common and its extent has been associated with increased joint pain and progression of disease. ${ }^{6,7}$ Therefore, many people assume that targeting synovial inflammation will improve pain and outcomes. However, evidence is increasing that antisynovial agents are not effective in various types of joint site osteoarthritis, from hydroxychloroquine to anti-tumour necrosis factor and antiinterleukin (IL) 1.5,89 Many argue this lack of efficacy is due to disease heterogeneity, but even studies that select individuals with high synovial inflammation have not shown efficacy. ${ }^{9}$ The association of synovial inflammation with the disease might be just that, an association. None of these observations relating to synovial inflammation show causality and they might just be a bystander or secondary phenomena of a disease-causing process in the joint, rather than a true treatment target.

How do we find effective therapies for symptomatic osteoarthritis? In many studies, including the study by van den Ende and colleagues, all patients had established osteoarthritis. Are we intervening too late and need to adopt the rheumatoid arthritis model, intervening early in those at risk? Or perhaps the lesson is that interventions must be better rooted in mechanistic evidence. Such evidence arguably needs to be established first in diseaserelevant preclinical models and experimental medicine studies before validation in disease-relevant preclinical models and experimental medicine studies, rather than just basing trials on clinical assumptions that treatments might work.

No one wants to see an erosion of therapeutic options for osteoarthritis, but equally important is to ensure
Lancet Rheumatol 2019 
that we do not hold on to or advocate treatments with associated cost and safety implications without adequate evidence for effect. Studies like this are therefore highly valuable, and should change our practice; as clinicians we must be agnostic and respond to evidence around us.

Key messages from this Article are that evidence is lacking to support use of low-dose radiation therapy for management of either hand or knee osteoarthritis. We should arguably turn our attentions to the identification of new treatments. Meanwhile, several evidencebased recommendations exist for management of hand and knee osteoarthritis-eg, being clear on diagnosis, giving positive messages with education, optimising bodyweight, exercise, and offloading of affected joints at their centre. ${ }^{10}$ Although much is left to do in the field, practitioners should continue to recommend therapies to their patients that have the best supporting evidence.

\section{Fiona E Watt}

Centre for Osteoarthritis Pathogenesis Versus Arthritis, Kennedy Institute of Rheumatology, NDORMS, University of Oxford, Oxford, OX3 7FY, UK fiona.watt@kennedy.ox.ac.uk

I work in the Centre for Osteoarthritis Pathogenesis Versus Arthritis and am a UK Research and Innovation Future Leaders Fellow with a research interest in the development of new treatments for osteoarthritis.
Minten MJM, Leseman-Hoogenboom MM, Kloppenburg M, et al. Lack of beneficial effects of low-dose radiation therapy on hand osteoarthritis symptoms and inflammation: a randomised, blinded, sham-controlled trial. Osteoarthritis Cartilage 2018; 26: 1283-90.

2 Mahler EAM, Minten MJ, Leseman-Hoogenboom MM, et al. Effectiveness of low-dose radiation therapy on symptoms in patients with knee osteoarthritis: a randomised, double-blinded, sham-controlled trial. Ann Rheum Dis 2019; 78: 83-90.

3 van den Ende CHM, Minten MJM, Leseman-Hoogenboom MM, et al. Long-term efficacy of low-dose radiation therapy on symptoms in patients with knee and hand osteoarthritis: follow-up results of two parallel randomised, sham-controlled trials. Lancet Rheumatol 2019; 1: XX-XX

4 Zhang W, Robertson J, Jones AC, Dieppe PA, Doherty M. The placebo effect and its determinants in osteoarthritis: meta-analysis of randomised controlled trials. Ann Rheum Dis 2008; 67: 1716-23.

5 Kingsbury SR, Tharmanathan P, Keding A, et al. hydroxychloroquine effectiveness in reducing symptoms of hand osteoarthritis: a randomized trial. Ann Intern Med 2018; 168: 385-95.

6 Baker K, Grainger A, Niu J, et al. Relation of synovitis to knee pain using contrast-enhanced MRIs. Ann Rheum Dis 2010; 69: 1779-83.

7 Atukorala I, Kwoh CK, Guermazi A, et al. Synovitis in knee osteoarthritis: a precursor of disease? Ann Rheum Dis 2016; 75: 390-95

8 Kloppenburg M, Peterfy C, Haugen IK, et al. Phase lla, placebo-controlled, randomised study of lutikizumab, an anti-interleukin-1 $\alpha$ and anti-interleukin-1 $\beta$ dual variable domain immunoglobulin, in patients with erosive hand osteoarthritis. Ann Rheum Dis 2019; 78: 413-20.

9 Kloppenburg M, Ramonda R, Bobacz K, et al. Etanercept in patients with inflammatory hand osteoarthritis (EHOA): a multicentre, randomised double-blind, placebo-controlled trial. Ann Rheum Dis 2018; 77: 1757-64

10 Bannuru RR, Osani MC, Vaysbrot EE, et al. OARSI guidelines for the non-surgical management of knee, hip, and polyarticular osteoarthritis. Osteoarthritis Cartilage 2019; 27: 1578-89. 